\title{
Development of the platform for Marine Early Warning Products Service based on Online ArcGIS
}

\author{
Yixin Huang ${ }^{1,2}$, Yonghua Sun ${ }^{1,2}$, Hao $\mathrm{Wu}^{1,2}$, Cankun Yang ${ }^{1,2}$ \\ ${ }^{1}$ State Key Laboratory Incubation Base of Urban Environmental Processes and Digital Simulation, \\ Capital Normal University, Beijing, 100048, China \\ ${ }^{2}$ College of Resources Environment \& Tourism, Capital Normal University, Beijing, 100048, China \\ email:emmayixin@163.com
}

Keywords: Marine early warning; Database index; Automatic template mapping

\begin{abstract}
The Clue of this paper is the data of marine environmental early warning service platform, and our research basis includes data classification, database index table, structure design and data consistence. The normalized data which is realized by ETL is ported in automatic drawing template, and use the pre configured symbol library, color library and pixel library to the marine environment early warning signs product data to automate the project, in order to realize the no manual intervention of the marine environmental warning signs product automatic template mapping. And then we release the marine environmental early warning products to achieve our goal which is the process of data batch input and output by ArcGIS Online based marine environment early warning product release database.
\end{abstract}

\section{Introduction}

In China, the coastal area is able to become the country's most economically developed regions, there is a factor cannot be ignored---the ocean, which not only provides a very rich natural resources for the coastal areas, but also creates a favorable climate environment as well as a convenient and efficient international access.

In the last 20 years, China's marine industry is thriving. As the most important growth point of China's economic development in the present and in the future, the marine economy is much higher than that of the coastal zone. However, any coin has two sides. The rapid development of coastal zone and marine economy has intensified the economic loss rate of marine disasters. Therefore, as a kind of effective technology to deal with the marine disasters, marine disaster early warning platform should be studied urgently.

\section{The standardization and database design of marine environmental data}

Marine environmental data as the core of marine warning, there are significant differences between its type and format. It has multi service, multi time, multi domain, mass and other characteristics. Based on the relevant standards and norms, this paper studies the database classification system of marine environmental warning system. The information classification method is applied to the database. A unified database naming standard is formulated for the platform of marine environmental warning system, and the multi - ocean data are normalized. On the basis of this, the design of the platform of the marine environmental warning system is carried out.

\section{The analysis of data format}

In the framework of the spatial database of marine early warning, it can be divided into three main parts, the basic database, the intermediate database and the published database. The product database of marine environmental warning involving a total of 8 kinds of special forecasting data, which consist of the wind field module group, sediment module group, rescue module group , temperature and salt flow module group and oil spill module, storm surge module group, coastal 
wave module group, saline module group. The wind field data segments subdivide 4 data products in details: 10 meters sea surface wind and 2 meters temperature and 2 meters sea wet and pressure. The data of the wind field data and the temperature and salt flow data are also stratified.

\section{The detailed design of database}

The product database of the marine environmental warning system is divided into 8 data sets into three databases, which are the basis database, the intermediate database and the published database. The basic database contains 8 data sets, including 4 types of data: Shapfile, Tin, Contour Line, Raster, the published database contains 3 types of data: MXD, GIF, BMP. The following is the relationship between the various databases:

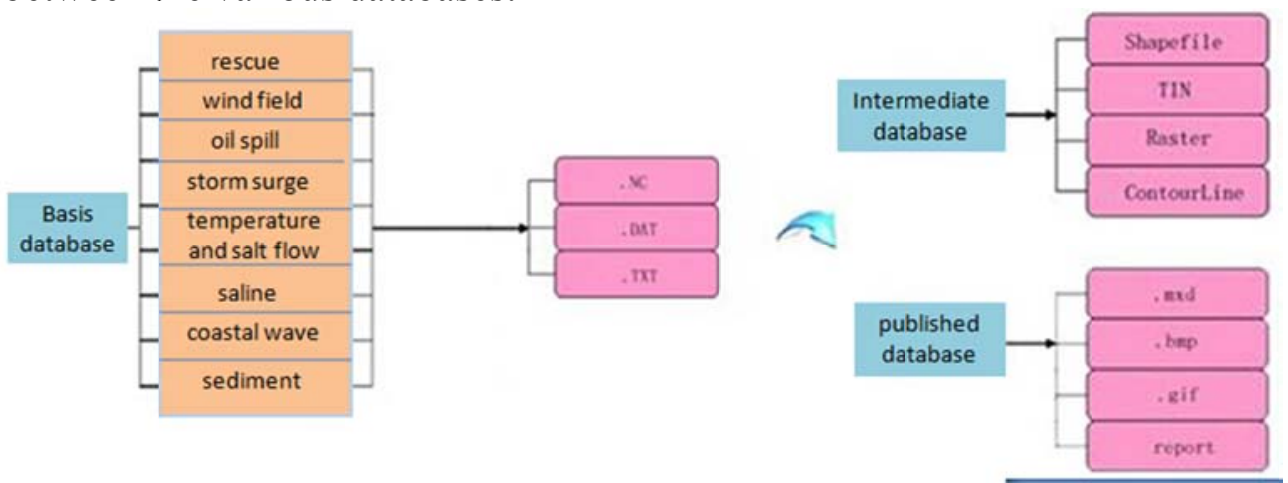

Fig.1. The relationship between the databases

\section{The ETL of marine warning products automation mapping}

ETL is the process of batch operation by extracting, converting and loading data. ETL for different databases, take different data extraction methods, and use a series of data conversion to achieve the data from the business model to the analyze model of the conversion. Through the built-in library functions, custom scripts or other extension methods, to achieve a variety of complex conversion, and support debug environment, can clearly monitor the data conversion; The theme of the data integration is extracting the multi-dimensional, multi spatial subject from the data source, and then extract, transform, and ultimately load to the target database [2]. The process can be represented in Figure 4.

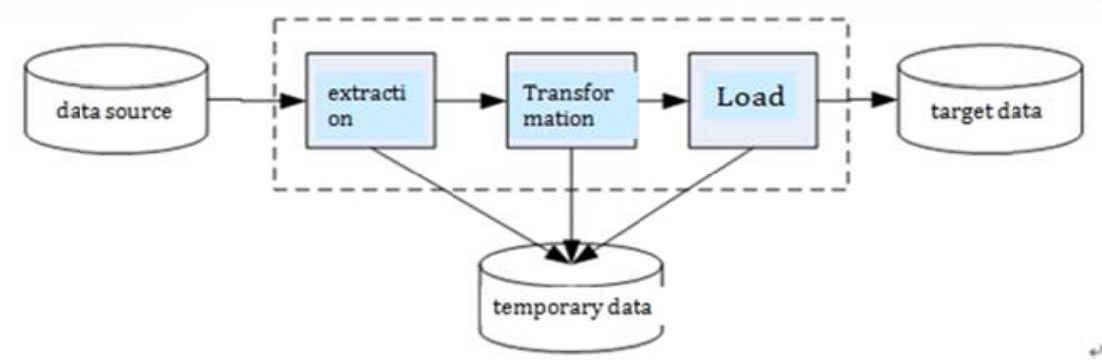

Fig.2. The simplification of data integration process

\section{Data extraction}

Data extraction means that table data is extracted from a single or multiple table data.

\section{Data conversion}

The essence of data conversion on the extracted data table is batch editing, formed to meet the special needs of variable field mapping.

(1) The representation method of the unified attribute value

There are different representations for the same attribute values in different data tables. Such as early warning product name field, and some form of field content as "longitude", "latitude", "depth", 
according to the needs of the mapping expression can be transformed into a unified data.

(2) Model calculation

To calculate the field value according to the professional model formula, probably including the addition and subtraction multiplication, or combination operation. Field value added: add the value of two or more fields, and store the result in a new field. Field value subtraction: subtraction value will be two or more fields, the results are stored into new fields. The field value is multiplied by two or more fields, and the result is stored in the new field.

(3)Table transposition

The transposition is a process of rows, columns, rows, to form the transpose is generally used for drawing the thematic variable as separate column expression did not exist, but exist in the line, cannot use a separate column mapping, it must be through the exchange of rows and columns of table transpose, which will attribute the thematic map return to stored in the column.

(4) Data cleansing

In the process of data conversion, a series of redundant data is generated, which is not special variable data, the data volume is increased, and the operating efficiency is reduced. Data cleaning is to remove the redundant data in the attribute table, and only the data of the special variables are kept. This data cleaning needs to be repeated, and the loss of useful data should be avoided.

\section{The template of the marine environment early warning rapid mapping}

After the extraction and conversion of the results of the data in accordance with the product data model organization requirements, stored in the database of the data, in order to provide a pre alarm special product use.

\section{Specifications of the marine warning product data model}

The data is extracted from the upper section of the paper, which is processed and loaded into the standard model of the marine environment. The model can be abstracted as follows:

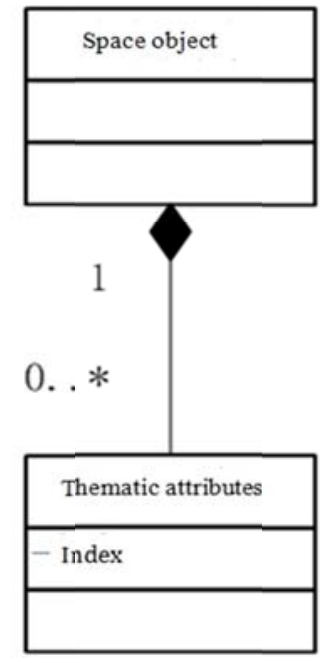

Fig.3. The Specification of Product data model

As the input specification subject data, the storage requirements and performance of the product are as follows:

\begin{tabular}{|l|l|l|l|l|}
\hline & Index field & (Time)constraint 1 & (Time)constraint 2 & $\ldots \ldots$ \\
\hline $\begin{array}{l}<\text { spatial } \\
\text { information }>\end{array}$ & $\begin{array}{l}\text { Expression } \\
\text { unit }\end{array}$ & Subject variable 1 & Subject variable 2 & $\ldots \ldots$ \\
\hline & $\ldots \ldots$ & $\ldots \ldots$ & $\ldots \ldots$ & $\ldots \ldots$ \\
\hline
\end{tabular}




\section{Template thematic chart rapid mapping technology}

In this paper, we consider the requirement of system intelligence, service and data storage, and use the ETL data processing technology as the basis of the volume automatic mapping technology. Through this technology, users can make a prediction of the time to be able to carry on the forecast map the automatic production, greatly simplifies the system uses the complexity and reduces the use process in the human error. Specific mapping techniques are as follows:

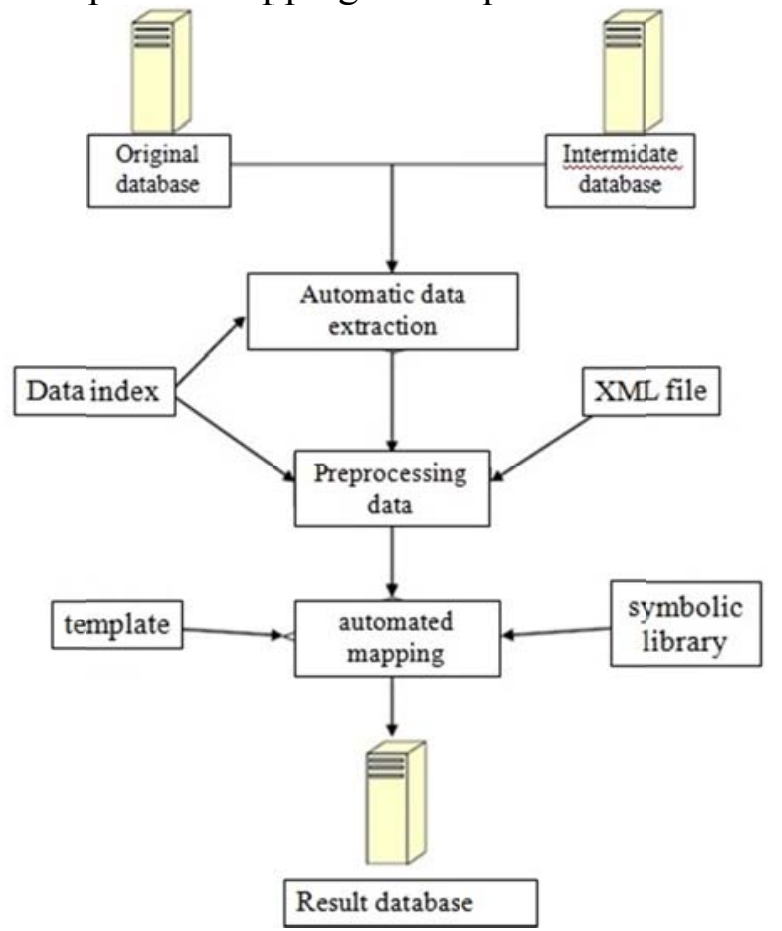

Fig.4. Automatic drawing flow chart

\section{The Online ArcGIS Map Publishing Library technology route}

In this paper, the $\mathrm{B} / \mathrm{S}$ model is used to construct the ocean disaster information management system, which uses ArcSDE to bulk storage in Oracle database. The data is processed by the model base, then the service is registered in Online ArcGIS and the data is distributed, thus providing data analysis and other functions through the Online ArcGIS powerful function. The specific technical flow chart is as follows: [6 8]:

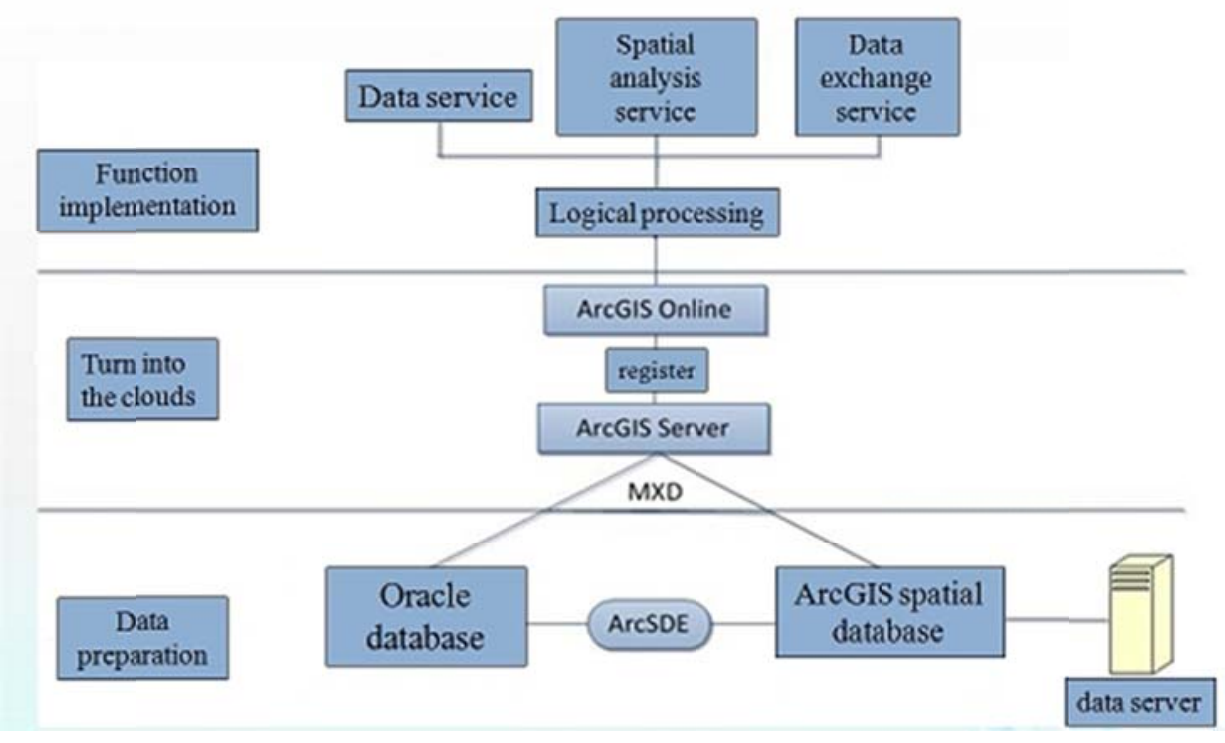




\section{Conclusions}

In this paper, a kind of marine environmental early warning data is proposed, which is based on the characteristics of multi species, multi diversified, multi phase and mass of marine data. And the paper presents a unified data naming standard of marine environmental warning system, and the design of the database of marine environmental warning system. This research divides the marine environment database into three parts, the basic database, the intermediate database and the published database.

In view of the current situation of product data content and data quantity increasing, this paper puts forward a kind of automatic drawing technology of marine early warning and forecasting, which is based on the data of marine environmental forecasting. Through ETL technology, this paper extracts, transforms and loads into the data processing, forming a subject oriented data conversion and integration technology, and through the parametric template library mapping and parametric mapping technology to complete the marine environmental forecasting data integrated automatic mapping.

\section{Acknowledgements}

In this paper, the research was sponsored by the Special Fund for Ocean Research in the Public Interest (Project No. 201205006-1) and (Project No. 201205017-6).

\section{References}

[1] Huaxi. High risk prediction of marine disaster in China [J]. marine development and management, 2010, 27 (7): 92-96.

[2] Zhou Aiwu, Zhang Zhihong. Comparison and analysis of two methods of data source integrated retrieval system [J]. information journal, 2007, 26 (1): 64-66.

[3] Deng Su, Zhou Maowei, Huang Hongbin. Design and implementation of metadata based ETL tool for [J]. science and technology and engineering, 6, 2006 (21): 3503-3507.

[4] Bian Fu Ling, Mei Kun. The location of construction of ontology [J]. geographic information world, geographical knowledge base 2008, 6 (4): 27-32.

[5] Wang Wu. Research and design of data cleaning method and tool design [D]. Shanghai Jiao Tong University, 2009

[6] ArcGIS and Oracle Spatial. To the plum city spatial database system based on [J]. engineering of Surveying and mapping, 2008, 17 (4): 70-73.

[7] Li Xiangyang, Wu Congcong, Lu Xiaoping, et al. Design and implementation of geological hazard information management system for $\mathrm{B} / \mathrm{S}$ structure $[\mathrm{J}]$. mapping and spatial geographic information, 36, 2013 (8): 23-25. 